
\title{
$\begin{array}{ll}\text { Research Square } & \begin{array}{l}\text { Preprints are preliminary reports that have not undergone peer review. } \\ \text { They should not be considered conclusive, used to inform clinical practice, } \\ \text { or referenced by the media as validated information. }\end{array}\end{array}$
}

\section{Effect of Body Mass Index on the Association Between Alcohol Consumption and the Development of Chronic Kidney Disease}

\author{
Yusaku Hashimoto \\ Department of Nephrology, Nagoya University Graduate School of Medicine \\ Sawako Kato \\ Department of Nephrology, Nagoya University Graduate School of Medicine \\ Yoshinari Yasuda \\ Department of Nephrology, Nagoya University Graduate School of Medicine \\ Takuji Ishimoto \\ Department of Nephrology, Nagoya University Graduate School of Medicine \\ Hiroaki Kawashiri \\ Takayama City Hall Public Health Department \\ Akihiro Hori \\ Kumiai Kosei Hospital \\ Takahiro Imaizumi \\ Department of Nephrology, Nagoya University Graduate School of Medicine \\ Shoichi Maruyama ( $\nabla$ marus@med.nagoya-u.ac.jp) \\ Department of Nephrology, Nagoya University Graduate School of Medicine
}

\section{Research Article}

Keywords: chronic kidney disease, alcohol consumption, body mass index

Posted Date: June 7th, 2021

DOI: https://doi.org/10.21203/rs.3.rs-569097/v1

License: () (1) This work is licensed under a Creative Commons Attribution 4.0 International License. Read Full License 


\section{Abstract}

Although previous studies demonstrated that alcohol consumption is associated with low chronic kidney disease (CKD) risk, they did not consider individual body mass and metabolic capacity. We examined whether the body mass index (BMI) affects the association between drinking and CKD. We defined CKD as an estimated glomerular filtration rate decline $<60 \mathrm{~mL} / \mathrm{min} / 1.73 \mathrm{~m}^{2}$ and/or dipstick proteinuria ( $\geq 1+$ ). Participants were 11,175 Japanese individuals aged 40-74 years without baseline CKD who underwent annual health checkups. Daily alcohol consumption was estimated from a questionnaire, and the participants were categorized as "infrequent drinkers," "light drinkers (< $20 \mathrm{~g} /$ day)," "moderate drinkers (20-40 g/day)," and "heavy drinkers ( $\geq 40 \mathrm{~g} /$ day)." Over a median 5-year observation period, 936 participants developed CKD. Cox proportional hazards models revealed that light drinkers had a significantly reduced risk of CKD compared with infrequent drinkers $(P=0.01)$. Stratified by BMI, the low BMI $\left(<18.5 \mathrm{~kg} / \mathrm{m}^{2}\right) \mathrm{group}$ had an increased risk of CKD even in light drinkers, while the high BMI $\left(\geq 25.0 \mathrm{~kg} / \mathrm{m}^{2}\right)$ group had a decreased risk of CKD regardless of alcohol consumption. Taken together, alcohol consumption did not reduce the CKD risk in all populations; individual tolerance must be considered.

\section{Introduction}

Chronic kidney disease (CKD) with proteinuria and/or a lowered glomerular filtration rate (GFR), is a growing public health issue worldwide. ${ }^{1,2}$ The number of CKD patients in Japan is 13.3 million, or $13 \%$ of the adult population. ${ }^{3}$ CKD is an important condition as it is not only a risk factor for progression to end-stage kidney disease but also for the development of cardiovascular disease (CVD) and all-cause mortality. ${ }^{4-8}$

It is important to pay attention to the modifiable risk factors such as lifestyle to prevent CKD development. ${ }^{9}$ A systematic review identified modifiable lifestyle factors, including alcohol consumption, for the primary prevention of CKD. ${ }^{10}$ This association was also observed across the entire range of alcohol consumption, with higher alcohol consumption associated with a lower risk of developing CKD. However, the systematic review demonstrated moderate heterogeneity, which suggested an inconsistent association between the amount of alcohol consumed and the risk of developing CKD. One of the studies in the systematic review showed that the risk of developing CKD decreased only in the group with an alcohol consumption $<20 \mathrm{~g} /$ day compared to that in the non-alcohol consuming group. ${ }^{11}$ Moreover, recent cohort studies have shown that high alcohol consumption ( $\geq 60 \mathrm{~g} / \mathrm{day}$ or $\geq$ $69.1 \mathrm{~g} /$ day) was associated with new-onset proteinuria. ${ }^{12,13}$ However, most previous studies did not examine the association between alcohol consumption and CKD development relative to alcohol tolerance in each individual, which may have led to inconsistent results.

The incidence of hypertension (HT) and diabetes mellitus (DM), which are risk factors for CKD, has been reported to show a U-shaped or linear association with alcohol consumption. ${ }^{14-17}$ Recent studies in Japan have shown that a higher body mass index (BMI) attenuates the risk of developing HT and DM due to alcohol consumption. ${ }^{18-20}$ However, no previous studies have examined this association with regard to CKD development. Therefore, we hypothesized that a high BMI would have a protective effect against CKD development and a lower BMI would enhance the harmful effects of alcohol consumption on CKD development.

This study aimed to examine whether alcohol intake is associated with CKD development in the general population and whether BMI modifies the association between alcohol consumption and the risk of CKD using data from health checkups.

\section{Results}

\section{Subject population}

In this cohort, we enrolled 19,950 participants who underwent health checkups and excluded 4524 participants who underwent only one health examination: 2,332 with prevalent CKD (estimated GFR [eGFR] $60 \mathrm{~mL} / \mathrm{min} / 1.73 \mathrm{~m}^{2}$ or the presence of proteinuria) at baseline; 1,046 who had CVD, chronic obstructive pulmonary disease (COPD), or liver disease; and 873 who had missing or inadequate data on alcohol drinking habits. CVD was defined as any of the following: myocardial infarction, coronary revascularization, heart failure, or stroke. Finally, the analytic cohort comprised 11,175 participants (Figure 1).

\section{Baseline characteristics}

Baseline characteristics according to alcohol consumption status are shown in Table 1. The median age was 62 years (interquartile range [IQR], 55-67 years). Compared to infrequent drinkers, the rest of the participants were more likely to be male, smokers, have higher blood pressure (BP), higher triglyceride (TG), and higher high-density lipoprotein cholesterol (HDL-C) levels, and lower low-density lipoprotein cholesterol (LDL-C) levels. During a median follow-up of 5 years (IQR, 2.9-7.6 years), there was a median of 5 visits (IQR, 3-8 visits), and a total of 936 patients developed CKD. Most participants underwent annual checkups, and the frequency of checkups was similar across groups.

\section{Alcohol consumption and CKD development}

Table 2 shows the association between alcohol consumption and CKD development. The multivariable-adjusted hazard ratios (HRs) (95\% confidence intervals; Cls) for CKD development were 0.81 (0.69-0.95) for light, 0.81 (0.65-1.00) for moderate, and 0.96 (0.74-1.23) for heavy drinkers, compared with infrequent drinkers as a reference. No significant trend was observed across categories of alcohol consumption ( $p=0.18$ for trend). Regarding eGFR decline, alcohol consumption had no significant effect on the outcome. Although not significant, light and moderate drinkers were less likely to have an eGFR decline with multivariable-adjusted HRs of 0.88 (95\% $\mathrm{Cl}, 0.69-1.12)$ and $0.92(95 \% \mathrm{Cl}, 0.62-1.34)$, respectively. Regarding proteinuria, light and 
moderate drinkers had a lower risk with multivariable-adjusted HRs of 0.73 (95\% Cls, $0.60-0.90)$ and $0.73(95 \% \mathrm{Cl}, 0.56-0.94)$, respectively. The risk of developing proteinuria showed a significant inverse trend with an increase in alcohol consumption $(p=0.034$ for trend).

\section{Association between alcohol consumption and CKD stratified by BMI}

Table 3 shows the association between alcohol consumption and CKD development stratified by $\mathrm{BMI}$. In the high $\mathrm{BMI}$ group (BMI $\left.\geq 25 \mathrm{~kg} / \mathrm{m}^{2}\right)$, the reduction in risk of CKD with alcohol consumption was more remarkable, with multivariable-adjusted $\mathrm{HRs}(95 \% \mathrm{Cl})$ of 0.80 (0.57-1.11) for light drinkers, $0.63(0.39-1.04)$ for moderate drinkers, and $0.61(0.34-1.11)$ for heavy drinkers. There was a significant inverse trend of decreasing CKD risk with increasing alcohol consumption ( $p=0.028$ for trend). In contrast, higher HRs for risk of CKD with alcohol consumption were observed in the low BMI group (BMI < $\left.18.5 \mathrm{~kg} / \mathrm{m}^{2}\right)$ group. The multivariable-adjusted HRs $(95 \% \mathrm{Cls})$ were $1.26(0.66-2.40)$ for light, 3.44 (1.60-7.42) for moderate, and 3.21 (1.23-8.37) for heavy drinkers. The risk of developing CKD showed a significant trend with an increase in alcohol consumption ( $p=0.003$ for trend). The association between alcohol consumption and the risk of CKD was modified by BMI ( $p=0.005$ for interaction). We also performed a sensitivity analysis using a different definition of alcohol consumption. This definition was based on both the frequency of drinking and the volume of alcohol per consumption. As in the main analysis, the risk of CKD increased with increasing drinking frequency and volume of alcohol per consumption in the low BMI group, while the reverse was noted in the high BMI group (Supplementary Table S1)).

\section{Discussion}

In this study, we demonstrated that light drinking was associated with a lower risk of CKD, and this association was modified by the BMI status. When the BMI was low, alcohol consumption was associated with a higher risk of CKD. On contrary, when the BMI was high, even heavy alcohol consumption was associated with a lower risk of CKD. These results suggest that a high BMI counteracts the harmful effects of alcohol on kidney function. To the best of our knowledge, our study is the first to evaluate the effect of BMI on the association between alcohol consumption and CKD development.

Our findings on the dose-response association between alcohol consumption and CKD development are partially consistent with those of previous studies. A recent systematic review and two large prospective cohort studies showed that alcohol consumption was associated with a lower risk of CKD regardless of the amount of alcohol consumption. ${ }^{10,21,22}$ Contrarily, in a large prospective study in Japan, only the light alcohol consumption group (<20 $\mathrm{g} /$ day) was associated with a lower risk of CKD. ${ }^{11}$ This discrepancy may be due to differences in the background of the target population. Previous epidemiological studies have reported that heavy alcohol consumption was associated with a higher incidence of HT and DM, which are known risk factors for CKD, but the alcohol-induced risk was reduced in those with higher BMI. ${ }^{14-20}$ However, minimal evidence is available on the association between alcohol consumption and the risk of CKD considering the effect of factors such as body mass on alcohol tolerance.

There are several possible mechanisms by which alcohol consumption may affect renal function, including protective ${ }^{10}$ and harmful effects. ${ }^{23,24}$ Regarding protective effects, clinical studies have shown that moderate alcohol intake prevents atherosclerosis via alcohol-induced changes in lipid profile and inflammation. ${ }^{25}$ Besides, animal studies have reported that low concentrations of alcohol protect podocytes, one of the key structures in the kidney, via acetaldehyde dehydrogenase and 20-hydroxyeicosatetraenoic acid. ${ }^{26}$ Furthermore, alcohol consumption has been reported to improve insulin sensitivity. ${ }^{27,28}$ Another animal study reported that podocytes are insulin sensitive and that this insulin sensitivity is important for maintaining the glomerular filtration barrier. ${ }^{29}$ Regarding harmful effects, excessive alcohol consumption has been reported to have a negative effect on the risk of atherosclerosis. ${ }^{30}$ Moreover, animal studies have reported that a high ethanol concentration significantly increases superoxide production in podocytes, resulting in increased oxidative stress and damage to podocytes. ${ }^{26}$ In addition, excessive alcohol consumption can cause liver damage, cirrhosis, and secondary renal damage. ${ }^{31,32}$ These mechanisms suggest that the harmful effects on renal function are more likely to occur when alcohol concentrations are high or when aldehydes, the metabolic intermediate products of alcohol, accumulate. These harmful effects are enhanced when alcohol is consumed beyond an individual's capacity; BMI is one of the factors that determine this capacity. ${ }^{33,34}$ In addition to body mass, genetic variations in the alcohol metabolism capacity should be considered in people with East Asian ancestry, including the Japanese populations. Mutations in the gene encoding the aldehyde dehydrogenase $2(A L D H 2)$ enzyme reduce acetaldehyde metabolism, predisposing to the flushing response. Individuals with mutations in the $A L D H 2$ gene are likely to have a lower $\mathrm{BMI}$ and to consume less alcohol, while those without these mutations tend to have a higher BMI. $^{35}$ Therefore, we considered that these mutations could partly account for the mechanism regarding the hypothesis that a high BMI would increase alcohol tolerance. A high BMI is still associated with alcohol tolerance, and our study demonstrated robust results regardless of the underlying mechanisms.

Our study has several limitations. First, we could not distinguish between individuals who had never consumed alcohol, those who consumed alcohol previously, and those who consumed alcohol occasionally at baseline, with all the above categorized as infrequent drinkers. The infrequent alcohol consumption category may include people who abstained from drinking due to ill health or pre-existing conditions. ${ }^{36}$ However, we excluded individuals with a history of CVD, COPD, liver disease, which may have resulted in alcohol cessation for health conditions. Second, this study defined the highest category of alcohol consumption as $\geq 40 \mathrm{~g} /$ day and the lowest category as $<20 \mathrm{~g} /$ day, which may not be sufficient to show a dose-response relationship between alcohol consumption and CKD. In previous studies examining the association between risk of CKD development and alcohol consumption with the highest consumption category being $60 \mathrm{~g}$ /day or more, the association was U-shaped or J-shaped. ${ }^{13,37}$ Conversely, when the highest consumption category was $30 \mathrm{~g}$ or $40 \mathrm{~g}$ /day or more, the risk decreased in a negative linear manner. ${ }^{22,38}$ In our study, overall, alcohol consumption and CKD risk were U-shaped, but there was a dose-dependent increase in risk in the low BMI group, a U-shape in the normal BMI group, and a dose-dependent decrease in risk in the high BMI group. Although it should be validated in a wider range of settings, we believe that stratification by BMI, which partly reflects an

Page $3 / 11$ 
individual's alcohol tolerance, will help examine the appropriate alcohol intake associated with CKD in this study. Third, we did not consider metabolizing enzymes such as alcohol dehydrogenase-1B and ALDH2. Almost all of the study participants were ethnic Japanese, and people with an Asian ancestry may be more sensitive to alcohol than those with a Caucasian ancestry due to genetic differences in alcohol-metabolizing enzymes, ${ }^{39}$ which may have affected their drinking habits. ${ }^{40}$ However, genetic tests are not widely available for health examination in the general population, nor is it routinely performed for risk stratification. Further studies are needed to evaluate the association between alcohol consumption and CKD development, considering genetic variations. Fourth, not all participants underwent continuous health checkups, and they may have not been tracked in the process due to moving to another municipality or death. However, we believe that bias is unlikely to occur in our cohort due to continuity in receiving annual checkups by most participants, the similarity in frequency and duration of follow-up across groups divided by drinking status, and the exclusion of individuals with preexisting conditions. Finally, this was an observational study; therefore, unmeasured confounding factors may exist, although we adjusted for clinically relevant confounders for CKD. However, the strength of the present study is that in our cohort, more than $50 \%$ of the people who were eligible for the specified health checkups had been receiving the checks since the program was started in 2008 . This percentage is higher than the national average of 30 to $35 \%$ for residents in other areas who are covered by the national health insurance scheme. ${ }^{41}$ Moreover, we have comprehensive lifestyle information, anthropometric measurements, and blood and urine test results for more than 10,000 participants, and these data are comparable to those of the National Database of Health Insurance Claims and the Specific Health Examination Database Open Data Japan, which is considered representative and meaningful. ${ }^{42}$

In conclusion, light alcohol consumption has beneficial effects on CKD development, and our findings underscore the effect modification of the association between alcohol consumption and CKD development by BMI. The benefits of alcohol consumption cannot be generalized and depend on an individual's constitution. Further study is needed to investigate the interaction between alcohol consumption and BMI for risk of CKD development in other ethnicities and age groups.

\section{Methods}

\section{Participant selection}

We conducted a cohort study, named the Prevention of Development of Chronic Kidney Disease-the PROMISE Study-to investigate the association between alcohol consumption and new-onset CKD. Eligible participants were those aged 40-74 years who underwent specific health checkups at Kumiai Welfare Hospital, Takayama City, Gifu Prefecture, Japan, from April 2008 to December 2016. In this study, a cohort was formed with participants enrolled in the residential national health insurance program. We obtained information regarding drinking and smoking habits, antihypertensive and diabetes medications, and medical history using self-administered questionnaires.

All participants were anonymous, and the study complied with the Declaration of Helsinki and the Ethical Guidelines for Epidemiological Research published by the Ministry of Education, Culture, Sports, Science and Technology, and the Ministry of Health, Labour and Welfare in Japan. The requirement for written informed consent was waived by Institutional Review Board Committee of Nagoya University Hospital (approval number: [20170004]) because the study participants did not undergo invasive procedures and only existing clinical data were collected.

\section{Definition of alcohol consumption}

Regarding alcohol consumption, the participants were asked how often how much sake (the standard drink in Japan) they consume per day. Daily alcohol consumption was assessed in units of 'gou' (a traditional Japanese unit of measurement) and subsequently converted to grams of alcohol per day. One unit (gou) of sake contains $20 \mathrm{~g}$ of alcohol and is equivalent to $500 \mathrm{~mL}$ of beer, $60 \mathrm{~mL}$ of whiskey, or $240 \mathrm{~mL}$ of wine. ${ }^{42,43}$ The following two questions were asked to confirm the drinking habit: " How often do you drink alcoholic beverages: every day/occasionally/ rarely or never ? " and "How many units of sake do you drink on the days you drink: $<1$ drink per day/1-2 drinks per day/2-3 drinks per day/ $\geq 3$ drinks per day?" Total alcohol consumption (in grams per day) was calculated from questions on the unit of sake consumed per day. We labeled the four categories as infrequent drinkers, light drinkers ( $<20 \mathrm{~g} /$ day), moderate drinkers (20 to $40 \mathrm{~g} /$ day), and heavy drinkers ( $\geq 40 \mathrm{~g} /$ day). Since it was not feasible to quantify the precise amount of alcohol consumed by participants who answered "occasionally," the participants who answered " rarely or never " and "occasionally" were grouped into the " infrequent drinkers " category for this analysis as the reference category. Some participants in the infrequent drinkers category may have refrained from drinking due to ill health or pre-existing conditions. ${ }^{36}$ Therefore, we excluded participants with a history of CVD, COPD, or liver diseases.

\section{Outcomes}

Outcomes were measured using data from each visit, even for those with intervals between visits. The outcome was CKD development, defined as a $25 \%$ decline in eGFR to less than $60 \mathrm{~mL} / \mathrm{min} / 1.73 \mathrm{~m}^{2}$ and/or a dipstick urinalysis protein score of $1+$ or greater (equivalent to $\geq 30 \mathrm{mg} / \mathrm{dL}$ ) during the followup period. ${ }^{44}$ Moreover, the effects of alcohol consumption on eGFR decline or proteinuria were investigated separately. To clarify whether the effects of alcohol consumption on the risk of CKD can be modified by the BMI status, we examined the association between alcohol consumption and the risk of CKD stratified by BMI.

\section{Physical examination and laboratory data}


Trained nurses measured the participants' BP using a standard protocol. After overnight fasting, venous blood was collected to measure the fasting plasma glucose, HbA1c, TG, HDL-C, LDL-C, and creatinine levels. Proteinuria was assessed using the dipstick method. eGFR was calculated using the formula of the Japanese Society of Nephrology [194 $\times$ age (year) $-0.287 \times$ serum creatinine $(\mathrm{mg} / \mathrm{dL})-1.094\left(\times 0.739\right.$ if female)]. ${ }^{45}$

\section{Other covariates}

Current smokers were defined as participants with any of the following habits: those who had been smoking since more than 6 months or more than 100 cigarettes in total and those who smoked in the last month. Others were categorized as non-smokers, including those who had a previous smoking habit. BMI was calculated by dividing the weight (in kilograms) by the square of the height (in meters). We divided BMI into three categories (< 18.5: low, 18.525.0: normal, and $\geq 25.0$ : high). HT was defined as any of the following: systolic BP $\geq 140 \mathrm{mmHg}$, diastolic BP $>90 \mathrm{mmHg}$, or use of antihypertensive medications. ${ }^{46}$ DM was defined as a fasting glucose level of $\geq 126 \mathrm{mg} / \mathrm{dL}, \mathrm{HbA} 1 \mathrm{c}$ (National Glycohemoglobin Standardization Program [NGSP]) level of $\geq 6.5 \%$, or receiving glucose-lowering therapy. ${ }^{47}$ After measuring the $\mathrm{HbA} 1 \mathrm{c}$ level (\%) in blood using the standard method proposed by the Japanese Diabetes Society (JDS), the NGSP equivalent was calculated using the following formula: HbA1c (NGSP) $=$ HbA1c (JDS) + 0.4. Dyslipidemia was defined as an LDL-C level $\geq 140 \mathrm{mg} / \mathrm{dL}$, an HDL-C level $<40 \mathrm{mg} / \mathrm{dL}$, a TG level $\geq 150 \mathrm{mg} / \mathrm{dL}$, or the use of lipid-lowering medication. ${ }^{48}$

\section{Statistical analysis}

Baseline characteristics are presented by category of alcohol consumption (infrequent, light, moderate, and heavy drinkers). The mean or median was calculated for continuous variables, and percentages were calculated for dichotomous variables. To examine the association between baseline alcohol consumption and CKD development, Cox proportional hazards models were employed to calculate the HRs and $95 \%$ Cls for each alcohol consumption category using infrequent drinkers as the reference category. Multivariable adjustments were performed as follows: age and sex (model 1 ) and age, sex, eGFR, BMI, the presence of HT, dyslipidemia, DM, and smoking status (current vs. noncurrent) (model 2). The proportional hazards assumption was tested using scaled Schoenfeld residuals. $\mathrm{P}$ for trend was derived from Cox proportional hazards models by treating alcohol consumption as a continuous linear term. Incidence rates were estimated using the person-year approach. We incorporated the interaction term between alcohol consumption and BMI categories into multivariable-adjusted models to assess the effect modification by BMI. P for interaction was derived using a likelihood ratio test from models with and without the cross-product term of alcohol category (infrequent, light, moderate, and heavy drinkers) and risk factors in the multivariable-adjusted model. All statistical analyses were performed using Stata version 16.0 MP (Stata Corp, www.stata.com). The statistical significance was set at $\mathrm{P}<0.05$.

\section{Declarations}

\section{Data availability}

The data that support the findings of this study are available from the corresponding author upon reasonable request.

\section{Acknowledgments}

We thank Yuya Itano and Shimon Kurasawa for their advice on statistical methods and data organization. We would also like to thank the staff of Kumiai Kosei Hospital and the staff of Takayama City Health Department for their cooperation in collecting the health checkup data. We would like to thank Editage (www.editage.com) for English language editing.

\section{Author contributions}

Y.H., T.I., and S.M. conceptualized and designed this study; H.K., A.K., S.K., and S.M. contributed to data collection and management; Y.H. and T.I. performed the formal analysis; Y.H. and T.I. wrote the manuscript with contributions from all authors. All authors discussed the results. All authors read and approved the final manuscript.

\section{Corresponding author}

Correspondence to Shoichi Maruyama and Takahiro Imaizumi.

\section{Competing interests}

The authors declare no competing interests.

\section{Funding}

This research did not receive any specific grant from funding agencies in the public, commercial, or not-for-profit sectors.

\section{Ethical standards}

We conducted the study in accordance with the guidelines of the Declaration of Helsinki. The institutional ethics committee of Nagoya University Graduate School of Medicine has formally approved the conduct of this study (No.2017-0004). Since the study data were provided anonymously, and the study participants did not receive any intervention, informed consent for study participation was not required. 


\section{References}

1. Jha, V. et al. Chronic kidney disease: Global dimension and perspectives. The Lancet382, 260-272 (2013).

2. Sherwood, M. \& Mccullough, P. A. Chronic kidney disease from screening, detection, and awareness, to prevention. Lancet Glob. Heal.4, e288-e289 (2016).

3. Imai, E. et al. Prevalence of chronic kidney disease in the Japanese general population. Clin. Exp. Nephrol.13,621-630 (2009).

4. Muntner, P., He, J., Hamm, L., Loria, C. \& Whelton, P. K. Renal insufficiency and subsequent death resulting from cardiovascular disease in the United States. J. Am. Soc. Nephrol.13, 745-53 (2002).

5. Anavekar, N. S. et al. Relation between Renal Dysfunction and Cardiovascular Outcomes after Myocardial Infarction. N. Engl. J. Med.351, 1285-1295 (2004).

6. Weiner, D. E. et al. Chronic Kidney Disease as a Risk Factor for Cardiovascular Disease and All-Cause Mortality: A Pooled Analysis of CommunityBased Studies. J. Am. Soc. Nephrol.15, 1307-1315 (2004).

7. Ninomiya, T. et al. Chronic kidney disease and cardiovascular disease in a general Japanese population: The Hisayama Study. Kidney Int.68, 228236 (2005).

8. Irie, F. et al. The relationships of proteinuria, serum creatinine, glomerular filtration rate with cardiovascular disease mortality in Japanese general population. Kidney Int.69, 1264-1271 (2006).

9. De Francisco, A. L. M., Fresnedo, G. F., Palomar, R., Piñera, C. \& Arias, M. The renal benefits of a healthy lifestyle. Kidney Int.68, 2-6 (2005).

10. Kelly, J. T. et al. Modifiable lifestyle factors for primary prevention of CKD: A systematic review and meta-analysis. J. Am. Soc. Nephrol.32, 239-253 (2021)..

11. Yamagata, K. et al. Risk factors for chronic kidney disease in a community-based population: A 10-year follow-up study. Kidney Int.71, 159-166 (2007).

12. Uehara, S. et al. Relationship between alcohol drinking pattern and risk of proteinuria: The Kansai Healthcare Study. J. Epidemiol.26, 464-470 (2016).

13. Kimura, Y. et al. Alcohol consumption and incidence of proteinuria: a retrospective cohort study. Clin. Exp. Nephrol.22, 1133-1142 (2018).

14. Okubo, Y. et al. Association of alcohol consumption with incident hypertension among middle-aged and older Japanese population: The Ibarakai prefectural health study (IPHS). Hypertension63, 41-47 (2014).

15. Roerecke, M. et al. The effect of a reduction in alcohol consumption on blood pressure: a systematic review and meta-analysis. Lancet Public Heal.2, e108-e120 (2017)

16. Taylor, B. et al. Alcohol and hypertension: Gender differences in dose-response relationships determined through systematic review and metaanalysis: REVIEW. Addiction104, 1981-1990 (2009).

17. Heianza, Y. et al. Role of alcohol drinking pattern in type 2 diabetes in Japanese men: the Toranomon Hospital Health Management Center Study 11 (TOPICS 11). Am. J. Clin. Nutr.97, 561-568 (2013).

18. Wakabayashi, I. Light-to-Moderate Alcohol Drinking Reduces the Impact of Obesity on the Risk of Diabetes Mellitus. J. Stud. Alcohol Drugs75, 10321038 (2014).

19. Nishigaki, D. et al. Body mass index modifies the association between frequency of alcohol consumption and incidence of hypertension in men but not in women: a retrospective cohort study. Hypertens. Res.43, 322-330 (2020).

20. Waki, K. et al. Alcohol consumption and other risk factors for self-reported diabetes among middle-aged Japanese: A population-based prospective study in the JPHC study cohort 1. Diabet. Med.22, 323-331 (2005).

21. Schaeffner, E. S. et al. Alcohol consumption and the risk of renal dysfunction in apparently healthy men. Arch. Intern. Med.165, 1048-1053 (2005).

22. Koning, S. H. et al. Alcohol consumption is inversely associated with the risk of developing chronic kidney disease. Kidney Int.87, 1009-1016 (2015).

23. Perneger, T. V, Whelton, P. K., Puddey, I. B. \& Klag, M. J. Risk of End-stage Renal Disease Associated with Alcohol Consumption. Am. J. Epidemlology150, 1275-81. (1999).

24. Shankar, A., Klein, R. \& Klein, B. E. K. The association among smoking, heavy drinking, and chronic kidney disease. Am. J. Epidemiol.164, 263-271 (2006).

25. Huang, Y. et al. Moderate alcohol consumption and atherosclerosis: Meta-analysis of effects on lipids and inflammation. Wien. Klin. Wochenschr.129, 835-843 (2017).

26. McCarthy, E. T. et al. Ethanol at low concentrations protects glomerular podocytes through alcohol dehydrogenase and 20-HETE. Prostaglandins Other Lipid Mediat.116-117, 88-98 (2015).

27. Davies, M. J. et al. Effects of moderate alcohol intake on fasting insulin and glucose concentrations and insulin sensitivity in postmenopausal women: A randomized controlled trial. J. Am. Med. Assoc.287, 2559-2562 (2002).

28. Paulson, Q. X., Hong, J., Holcomb, V. B. \& Nunez, N. P. Effects of body weight and alcohol consumption on insulin sensitivity. Nutr. J.9, 1-14 (2010).

29. Welsh, G. I. et al. Insulin signaling to the glomerular podocyte is critical for normal kidney function. Cell Metab.12, 329-340 (2010).

30. Kiechl, S. et al. Alcohol consumption and atherosclerosis: What is the relation?: Prospective results from the Bruneck Study. Stroke29, $900-907$ (1998) 
31. Rocco, A., Compare, D., Angrisani, D., Sanduzzi Zamparelli, M. \& Nardone, G. Alcoholic disease: Liver and beyond. World J Gastroenterol. 20, 146529(2014).

32. Janicko, M., Veseliny, E., Senajova, G. \& Jarcuska, P. Predictors of hepatorenal syndrome in alcoholic liver cirrhosis. Biomed. Pap.159, $661-665$ (2015).

33. Min Qi Wang, Nicholson, M. E., Jones, C. S., Fitzhugh, E. C. \& Westerfield, C. R. Acute alcohol intoxication, body composition, and pharmacokinetics. Pharmacol. Biochem. Behav.43, 641-643 (1992).

34. Maudens, K. E. et al. The influence of the body mass index (BMI) on the volume of distribution of ethanol. Forensic Sci. Int.243, 74-78 (2014).

35. Matoba, N. et al. GWAS of 165,084 Japanese individuals identified nine loci associated with dietary habits. Nat. Hum. Behav.4, 308-316 (2020).

36. Shaper, A. G., Wannamethee, G. \& Walker, M. Alcohol and mortality in British men: explaining the U-shaped curve. Lancet332, 1267-1273 (1988).

37. Kyoko, K. et al. Drinking Pattern and Risk of Chronic Kidney Disease: The Kansai Healthcare Study. Am J Nephro/40, 516-522 (2014).

38. White, S. L. et al. Alcohol consumption and 5-year onset of chronic kidney disease: the AusDiab study. Nephrol Dial Transpl. 2464-2472 (2009).

39. Goedde, H. W. et al. Distribution of ADH2 and ALDH2 genotypes in different populations. Hum. Genet.88, 344-346 (1992).

40. Matsuo, K. et al. Alcohol dehydrogenase 2 His47Arg polymorphism influences drinking habit independently of aldehyde dehydrogenase 2 Glu487Lys polymorphism: Analysis of 2,299 Japanese subjects. Cancer Epidemiol. Biomarkers Prev.15, 1009-1013 (2006).

41. The 4th NDB Open Data. Japan. https://www.mhlw.go.jp/stf/seisakunitsuite/bunya/0000177221_00003.html (2017).

42. Nagakura, Y., Kato, H., Asano, S., Jinno, Y. \& Tanei, S. The Significant Association between Health Examination Results and Population Health: A Cross-Sectional Ecological Study Using a Nation-Wide Health Checkup Database in Japan. Int. J. Environ. Res. Public Health18, 836 (2021).

43. Guo, R. \& Jun, R. Alcohol and acetaldehyde in public health: From marvel to menace. Int J Environ Res Public Health. 7, 1285-1301 (2010).

44. Levin, A. \& Stevens, P. E. Summary of KDIGO 2012 CKD Guideline: Behind the scenes, need for guidance, and a framework for moving forward. Kidney Internationa/85, 49-61 (2014).

45. Matsuo, S. et al. Revised Equations for Estimated GFR From Serum Creatinine in Japan. Am. J. Kidney Dis.53, 982-992 (2009).

46. Umemura, S. et al. The Japanese Society of Hypertension Guidelines for the Management of Hypertension (JSH 2019). Hypertension Research42, 1235-1481 (2019).

47. Seino, Y. et al. Report of the committee on the classification and diagnostic criteria of diabetes mellitus. J. Diabetes Investig.1, 212-228 (2010).

48. Teramoto, T. et al. Diagnosis of Atherosclerosis. J. Atheroscler. Thromb.21, 296-298 (2014).

\section{Tables}

Table1. Baseline characteristics stratified by drinking status 


\begin{tabular}{|c|c|c|c|c|c|}
\hline & & Infrequent & Light drinkers & Moderate drinkers & Heavy drinkers \\
\hline & Total & drinkers & $<20 \mathrm{~g} /$ day & 20 to $40 \mathrm{~g} /$ day & $\geq 40 \mathrm{~g} /$ day \\
\hline & $\mathrm{N}=11,175$ & $N=6,199$ & $N=3,157$ & $\mathrm{~N}=1,162$ & $N=657$ \\
\hline Age & $62(55-67)$ & $62(56-67)$ & $61(53-66)$ & $62(53-67)$ & $60(50-65)$ \\
\hline Sex male, $\mathrm{n}(\%)$ & $4,494(40 \%)$ & $1,368(22 \%)$ & $1,544(49 \%)$ & $985(85 \%)$ & $597(91 \%)$ \\
\hline Current smoker, $\mathrm{n}(\%)$ & $2,042(18 \%)$ & $787(13 \%)$ & $561(18 \%)$ & $393(34 \%)$ & $301(46 \%)$ \\
\hline $\operatorname{BMI}\left(\mathrm{kg} / \mathrm{m}^{2}\right)$, mean [SD] & 22.3 [3.1] & $22.2[3.2]$ & $22.4[2.9]$ & $22.7[2.9]$ & $22.6[2.9]$ \\
\hline$<18.5, \mathrm{n}(\%)$ & $994(8.9)$ & $674(10.9)$ & $222(7.0)$ & $56(4.8)$ & $42(6.4)$ \\
\hline $18.5-25, \mathrm{n}(\%)$ & $8,196(73.3)$ & $4,446(71.7)$ & $2,363(74.9)$ & $894(76.9)$ & $493(75.0)$ \\
\hline$\geq 25, \mathrm{n}(\%)$ & $1,985(17.8)$ & $1,079(17.4)$ & $572(18.1)$ & $212(18.2)$ & $122(18.6)$ \\
\hline Antihypertensive drug, $\mathrm{n}(\%)$ & $1,993(18 \%)$ & $982(16 \%)$ & $584(18 \%)$ & $254(22 \%)$ & $173(26 \%)$ \\
\hline Antihyperglycemic drug, n (\%) & $384(3 \%)$ & $228(4 \%)$ & $96(3 \%)$ & $39(3 \%)$ & $21(3 \%)$ \\
\hline Antihyperlipidemic drug, n (\%) & $1,190(11 \%)$ & $824(13 \%)$ & $275(9 \%)$ & $58(5 \%)$ & $33(5 \%)$ \\
\hline eGFR $\left(\mathrm{mL} / \mathrm{min} / 1.73 \mathrm{~m}^{2}\right)$, mean [SD] & 78 [12] & 77 [12] & 78 [12] & 79 [12] & 81 [12] \\
\hline Fasting blood glucose (mg/dL), mean [SD] & $92[18]$ & $92[17]$ & $92[17]$ & $95[17]$ & $95[19]$ \\
\hline $\mathrm{HbA} 1 \mathrm{c}(\%)$, mean [SD] & $5.7[0.5]$ & $5.8[0.6]$ & $5.7[0.5]$ & $5.7[0.7]$ & $5.6[0.7]$ \\
\hline LDL-C (mg/dL), mean [SD] & $119[30]$ & $123[29]$ & 118 [29] & 108 [29] & $102[31]$ \\
\hline $\mathrm{TG}(\mathrm{mg} / \mathrm{dL})$, median (IQR) & $92(67-132)$ & $92(68-128)$ & $90(65-130)$ & $96(68-144)$ & $111(74-175)$ \\
\hline $\mathrm{HDL}-\mathrm{C}(\mathrm{mg} / \mathrm{dL})$, mean [SD] & 60 [14] & 59 [14] & $61[14]$ & $62[15]$ & $63[16]$ \\
\hline Systolic BP (mmHg), mean [SD] & $125[18]$ & $123[17]$ & $125[18]$ & $130[19]$ & $132[18]$ \\
\hline Diastolic BP (mmHg), mean [SD] & $75[12]$ & $74[12]$ & $75[12]$ & $79[11]$ & $80[11]$ \\
\hline Incidence of CKD, n (\%) & $936(8.4)$ & $484(7.8)$ & $249(7.9)$ & $119(10.2)$ & $84(12.8)$ \\
\hline Follow up period (year), median (IQR) & $5.0(2.9-7.6)$ & $5.0(3.0-7.7)$ & $5.0(2.7-7.6)$ & $5.1(3.0-7.3)$ & $5.0(3.0-7.1)$ \\
\hline Number of checkups (visits), median (IQR) & $5.0(3.0-8.0)$ & $5.0(3.0-8.0)$ & $5.0(3.0-8.0)$ & $5.0(3.0-7.0)$ & $5.0(3.0-7.0)$ \\
\hline
\end{tabular}

Data are presented as number (\%) for categorical variables and mean [SD standard deviation] or median (IQR interquartile range) for continuous variables as appropriate.

BMI body mass index; eGFR estimated glomerular filtration rate; LDL-C low-density lipoprotein cholesterol; TG triglyceride; HDL-C high-density lipoprotein cholesterol; BP blood pressure; CKD chronic kidney disease.

Table 2. Result of the Cox proportional hazards model for the association between alcohol consumption and CKD development 


\begin{tabular}{|c|c|c|c|c|c|c|c|c|c|}
\hline \multirow[b]{2}{*}{ Amount of alcohol consumption } & \multirow{2}{*}{$\begin{array}{l}\text { Incidence rate (per } \\
1000 \mathrm{PY})\end{array}$} & \multicolumn{4}{|c|}{ Age and sex adjusted model 1} & \multicolumn{4}{|c|}{ Multivariate model $2^{a}$} \\
\hline & & HR & $95 \% \mathrm{Cl}$ & $\begin{array}{l}\mathrm{P} \\
\text { value }\end{array}$ & $\begin{array}{l}\mathrm{P} \\
\text { trend }^{\mathrm{b}}\end{array}$ & HR & $\begin{array}{l}95 \% \\
\mathrm{Cl}\end{array}$ & $\begin{array}{l}P \\
\text { value }\end{array}$ & $\begin{array}{l}\mathrm{P} \\
\text { trend }^{\mathrm{b}}\end{array}$ \\
\hline \multicolumn{10}{|l|}{ CKD } \\
\hline \multicolumn{10}{|l|}{$\begin{array}{l}\left(\mathrm{eGFR}<60 \mathrm{~mL} / \mathrm{min} / 1.73 \mathrm{~m}^{2}\right. \\
\text { and/or proteinuria) }\end{array}$} \\
\hline Infrequent & 16.2 & Ref. & & & 0.32 & Ref. & & & 0.18 \\
\hline$<20$ g/day & 16.6 & 0.81 & $\begin{array}{l}0.69- \\
0.95\end{array}$ & 0.01 & & 0.81 & $\begin{array}{l}0.69- \\
0.95\end{array}$ & 0.01 & \\
\hline $20-40$ g/day & 21.4 & 0.82 & $\begin{array}{l}0.66- \\
1.02\end{array}$ & 0.07 & & 0.81 & $\begin{array}{l}0.65- \\
1.00\end{array}$ & 0.05 & \\
\hline$\geq 40 \mathrm{~g} /$ day & 27.2 & 1.01 & $\begin{array}{l}0.79- \\
1.30\end{array}$ & 0.91 & & 0.96 & $\begin{array}{l}0.74- \\
1.23\end{array}$ & 0.74 & \\
\hline \multicolumn{10}{|l|}{$\begin{array}{l}\text { eGFR decline to }<60 \mathrm{~mL} / \mathrm{min} / 1.73 \\
\mathrm{~m}^{2}\end{array}$} \\
\hline Infrequent & 7.9 & Ref. & & & 0.32 & Ref. & & & 0.56 \\
\hline$<20 \mathrm{~g} /$ day & 6.5 & 0.90 & $\begin{array}{l}0.70- \\
1.15\end{array}$ & 0.39 & & 0.88 & $\begin{array}{l}0.69- \\
1.12\end{array}$ & 0.31 & \\
\hline $20-40 \mathrm{~g} /$ day & 6.4 & 0.96 & $\begin{array}{l}0.65- \\
1.41\end{array}$ & 0.84 & & 0.92 & $\begin{array}{l}0.62- \\
1.34\end{array}$ & 0.65 & \\
\hline$\geq 40 \mathrm{~g} /$ day & 9.3 & 1.55 & $\begin{array}{l}1.01- \\
2.36\end{array}$ & 0.04 & & 1.4 & $\begin{array}{l}0.91- \\
2.14\end{array}$ & 0.12 & \\
\hline \multicolumn{10}{|l|}{ New-onset of proteinuria } \\
\hline Infrequent & 9 & Ref. & & & 0.036 & Ref. & & & 0.034 \\
\hline$<20$ g/day & 10.2 & 0.72 & $\begin{array}{l}0.58- \\
0.88\end{array}$ & 0.001 & & 0.73 & $\begin{array}{l}0.60- \\
0.90\end{array}$ & 0.003 & \\
\hline $20-40$ g/day & 15 & 0.72 & $\begin{array}{l}0.56- \\
0.93\end{array}$ & 0.01 & & 0.73 & $\begin{array}{l}0.56- \\
0.94\end{array}$ & 0.02 & \\
\hline$\geq 40 \mathrm{~g} /$ day & 19.2 & 0.84 & $\begin{array}{l}0.63- \\
1.13\end{array}$ & 0.25 & & 0.82 & $\begin{array}{l}0.61- \\
1.11\end{array}$ & 0.21 & \\
\hline
\end{tabular}

Outcomes were CKD (composite outcome of eGFR decline and/or new-onset of proteinuria) and eGFR decline and new-onset of proteinuria, respectively. a Multivariable adjustment included age, sex, eGFR, hypertension, diabetes mellitus, hyper lipidemia, body mass index, smoking status. b P trend was derived from Cox proportional hazards regression models by treating alcohol consumption as a continuous linear term. CKD chronic kidney disease; eGFR estimated glomerular filtration rate; Cl confidence interval; HR hazard ratio; PY person-years.

Table 3. Result of the Cox proportional hazards model for the association between alcohol consumption and CKD development stratified by BMI 


\begin{tabular}{|c|c|c|c|c|c|c|c|c|c|c|c|c|}
\hline \multirow{3}{*}{$\begin{array}{l}\text { Amount of } \\
\text { alcohol } \\
\text { consumption }\end{array}$} & \multicolumn{4}{|l|}{ CKD } & \multicolumn{4}{|c|}{ eGFR decline to $<60 \mathrm{~mL} / \mathrm{min} / 1.73 \mathrm{~m}^{2}$} & \multicolumn{4}{|c|}{ New-onset of proteinuria } \\
\hline & \multicolumn{4}{|c|}{$\begin{array}{l}\text { Multivariate } \\
\text { model }^{\mathrm{a}}\end{array}$} & \multicolumn{4}{|c|}{$\begin{array}{l}\text { Multivariate } \\
\text { model }^{\mathrm{a}}\end{array}$} & \multicolumn{4}{|c|}{$\begin{array}{l}\text { Multivariate } \\
\text { model }^{a}\end{array}$} \\
\hline & HR & $\begin{array}{l}95 \% \\
\mathrm{Cl}\end{array}$ & $\begin{array}{l}\mathrm{P} \\
\text { trend }^{\mathrm{b}}\end{array}$ & $\begin{array}{l}\mathrm{P} \\
\text { interaction }^{\mathrm{c}}\end{array}$ & HR & $\begin{array}{l}95 \% \\
\mathrm{Cl}\end{array}$ & $\begin{array}{l}\mathrm{P} \\
\text { trend }^{\mathrm{b}}\end{array}$ & $\begin{array}{l}\mathrm{P} \\
\text { interaction }^{\mathrm{c}}\end{array}$ & HR & $\begin{array}{l}95 \% \\
\mathrm{Cl}\end{array}$ & $\begin{array}{l}\mathrm{P} \\
\text { trend }^{\mathrm{b}}\end{array}$ & $\begin{array}{l}\mathrm{P} \\
\text { interaction }^{\mathrm{c}}\end{array}$ \\
\hline $\mathrm{BMI}<18.5$ & & & & 0.005 & & & & 0.03 & & & & 0.03 \\
\hline Infrequent & Ref. & & 0.003 & & Ref. & & 0.054 & & Ref. & & 0.019 & \\
\hline$<20 \mathrm{~g} /$ day & 1.26 & $\begin{array}{l}0.66- \\
2.40\end{array}$ & & & 0.79 & $\begin{array}{l}0.28- \\
2.23\end{array}$ & & & 1.77 & $\begin{array}{l}0.76- \\
4.14\end{array}$ & & \\
\hline $20-40$ g/day & 3.44 & $\begin{array}{l}1.60- \\
7.42\end{array}$ & & & 1.94 & $\begin{array}{l}0.48- \\
7.81\end{array}$ & & & 4.58 & $\begin{array}{l}1.75- \\
12.0\end{array}$ & & \\
\hline$\geq 40 \mathrm{~g} /$ day & 3.21 & $\begin{array}{l}1.23- \\
8.37\end{array}$ & & & 5.86 & $\begin{array}{l}1.42- \\
24.2\end{array}$ & & & 2.66 & $\begin{array}{l}0.75- \\
9.40\end{array}$ & & \\
\hline \multicolumn{13}{|l|}{ BMI 18.5-25 } \\
\hline Infrequent & Ref. & & 0.17 & & Ref. & & 0.15 & & Ref. & & 0.013 & \\
\hline$<20 \mathrm{~g} /$ day & 0.78 & $\begin{array}{l}0.65- \\
0.95\end{array}$ & & & 0.96 & $\begin{array}{l}0.72- \\
1.27\end{array}$ & & & 0.67 & $\begin{array}{l}0.52- \\
0.86\end{array}$ & & \\
\hline $20-40 \mathrm{~g} /$ day & 0.75 & $\begin{array}{l}0.58- \\
0.98\end{array}$ & & & 1.14 & $\begin{array}{l}0.74- \\
1.78\end{array}$ & & & 0.61 & $\begin{array}{l}0.44- \\
0.83\end{array}$ & & \\
\hline$\geq 40 \mathrm{~g} /$ day & 0.96 & $\begin{array}{l}0.71- \\
1.29\end{array}$ & & & 1.65 & $\begin{array}{l}0.99- \\
2.74\end{array}$ & & & 0.78 & $\begin{array}{l}0.55- \\
1.11\end{array}$ & & \\
\hline \multicolumn{13}{|l|}{ BMI $25 \leq$} \\
\hline Infrequent & Ref. & & 0.028 & & Ref. & & 0.018 & & Ref. & & 0.16 & \\
\hline$<20$ g/day & 0.80 & $\begin{array}{l}0.57- \\
1.11\end{array}$ & & & 0.69 & $\begin{array}{l}0.40- \\
1.19\end{array}$ & & & 0.78 & $\begin{array}{l}0.52- \\
1.17\end{array}$ & & \\
\hline $20-40 \mathrm{~g} /$ day & 0.63 & $\begin{array}{l}0.39- \\
1.04\end{array}$ & & & 0.26 & $\begin{array}{l}0.08- \\
0.86\end{array}$ & & & 0.76 & $\begin{array}{l}0.44- \\
1.32\end{array}$ & & \\
\hline$\geq 40 \mathrm{~g} /$ day & 0.61 & $\begin{array}{l}0.34- \\
1.11\end{array}$ & & & 0.45 & $\begin{array}{l}0.13- \\
1.52\end{array}$ & & & 0.68 & $\begin{array}{l}0.36- \\
1.32\end{array}$ & & \\
\hline
\end{tabular}

Outcomes were CKD (composite outcome of eGFR decline and/or new-onset of proteinuria) and eGFR decline and new-onset of proteinuria, respectively.

a Multivariable adjustment included age, sex, eGFR, hypertension, diabetes mellitus, hyper lipidemia, smoking status.

b P trend was derived from Cox proportional hazards regression models by treating alcohol consumption as a continuous linear term.

c P interaction was derived by using a likelihood ratio test from models with and without the cross-product term of alcohol category (Infrequent, light, moderate, and heavy drinkers) and risk factor in the multivariable-adjusted model.

CKD chronic kidney disease; eGFR estimated glomerular filtration rate; BMI body mass index; Cl confidence interval; HR hazard ratio.

\section{Figures}


People who underwent the specific healthcheckups at Kumiai Kousei

Hospital, Gifu, Japan from April 2008 to December 2016.(n=19,950)

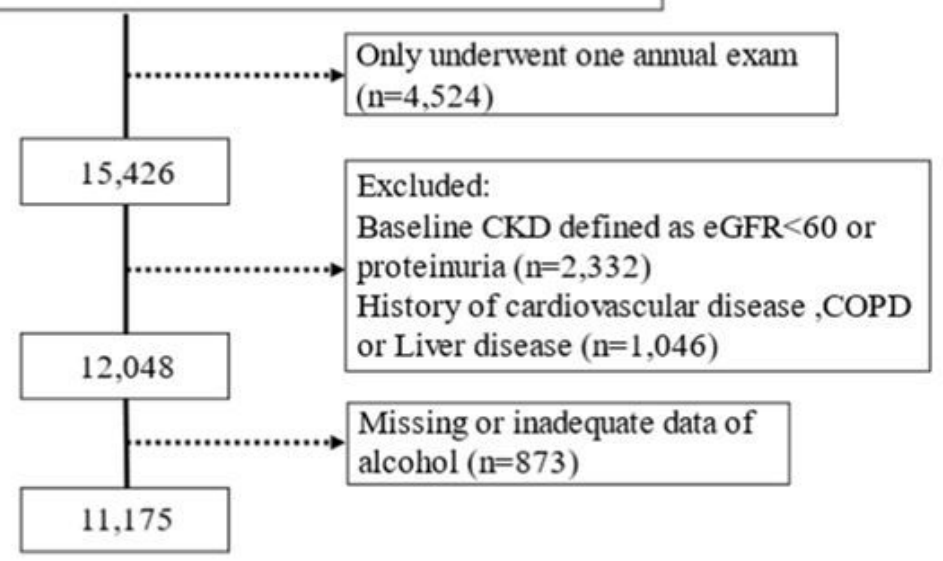

Figure 1

Flowchart of participant selection Between April 2008 and December 2016, 19,950 participants underwent health checkups at Kumiai Kosei Hospital, of which 15,426 participants who underwent medical checkups at least twice were included in the study. Patients with chronic kidney disease at the time of the first visit $(n=2,332)$ and those with a history of cardiovascular disease, chronic obstructive pulmonary disease, or liver disease $(n=1,046)$ were excluded, resulting in a total of 12,048 participants. Finally, 11,175 participants with sufficient information in the alcohol questionnaires were included in the analysis.

\section{Supplementary Files}

This is a list of supplementary files associated with this preprint. Click to download.

- Supplementary.docx 